\title{
USING A MIXED DEM/FEM APPROACH TO MODEL ADVANCED DAMAGE OF REINFORCED CONCRETE UNDER IMPACT
}

\author{
S. POTAPOV ${ }^{1}$, A. MASUREL ${ }^{1}$, L. DAUDEVILLE ${ }^{2} \&$ P. MARIN ${ }^{2}$ \\ ${ }^{1}$ IMSIA (UMR EDF-CNRS-CEA-ENSTA 9219) 1, Avenue du Général de Gaulle, 92141 Clamart Cedex, France \\ ${ }^{2}$ Laboratoire 3S-R (UMR 5521) Rue de la Piscine, BP 53, 38041 Grenoble Cedex 9, France.
}

\begin{abstract}
Advanced damage behaviour of reinforced concrete is modelled using a mixed modelling approach, in which concrete is represented through the spherical-type discrete element model, whereas steel reinforcement is modelled by using beam-type finite elements. An original steel-concrete bond model developed and calibrated on pull-out tests is used to ensure transfer of forces between steel and concrete. The proposed approach is applied to simulate soft and hard-type impacts on RC beams within a very complete modelling framework, thus allowing validating by comparison with experimental data the overall numerical approach developed.

Keywords: damage, discrete element method, impact, reinforced concrete, steel-concrete bond model.
\end{abstract}

\section{INTRODUCTION}

Reinforced concrete (RC) structures are widely used as shielding barriers to protect sensitive equipment of industrial facilities such as nuclear power plants. Those protective structures are devised to resist namely impacts of projectiles generated either by natural hazard phenomena such as tornados or by human induced events such as a deliberate airplane crash. Because of the extreme severity of such an accidental loading, assessment of the protective structures must go far beyond verification of the resistance to normal operating conditions: it is necessary to investigate the response of the structure until almost its complete failure to assess correctly its ultimate resistance capacity.

While continuous approaches such as the finite element method (FEM) are suitable for the nonlinear analysis of structures before failure, they reach their limits when trying to describe macro cracking and fragmentation mechanisms. Even if it is possible to generate discontinuities in the standard FEM model by using the element erosion technique proposed by Belytschko and Lin [1] and implemented in most commercial crash codes such as LS-DYNA or ABAQUS Explicit, the numerical precision of the resolution algorithm becomes difficult to control during the advanced fragmentation process due to difficulties of mass and energy conservation and of contact treatment of newly created non-smooth surfaces. Furthermore, numerical results are strongly dependent on the erosion criterion used, which explains why the FEM simulations with activated erosion struggle to produce quantitatively realistic and predictive results in the presence of strong material discontinuities.

The discrete element method (DEM) is a powerful alternative to FEM when advanced damage states and failure of concrete have to be studied. Indeed, DEM permits very easily the obtaining of realistic macro-crack patterns and material fragments due to its discontinuous 
nature. Most discrete approaches proposed in the literature study fracturing of rocks and plain concrete, i.e. the materials that possess homogeneous properties in an undamaged state. Thus, particle-based [2, 3], lattice-based [4], and mixed lattice discrete particle models [5] have been proposed to study material fracturing at different micro, meso or macroscopic modelling scales. At the micro and even meso level, concrete is a highly heterogeneous material, and its modelling needs a very complex modelling framework to describe different constituents and their interactions. While this kind of model possesses very good predictive capabilities under a wide variety of loading conditions [5] at the level of material samples, it remains difficult to apply to industrial size structures for the reasons of prohibitive computational cost and difficulty in visualizing and interpreting the simulation results. To study large-size structures or even entire buildings, only the macroscopic modelling scale seems suitable.

An important aspect to be dealt with when simulating failure of RC structures is to model the steel reinforcement and its interaction with concrete. Discrete approaches do it either by simply modifying local concrete properties or by incorporating explicitly the steel reinforcement within the model as in Kim et al. [6]. It should be noted that only explicit modelling of the reinforcement is reliable in such a context because it can represent correctly the fact that the reinforcement takes over in zones where concrete is completely fractured, thus maintaining the load carrying capacity of the structure.

This paper presents an original mixed DEM/FEM numerical approach able to simulate the response of industrial-size RC structures under severe impacts. A brief description of the spherical-type DEM, adopted herein to model cohesive and fracturing behaviour of concrete at macroscopic level, and the steel-concrete bond model is given. The proposed approach is applied then to simulate soft and hard-type impacts on RC beams within a very complete modelling framework, allowing us to validate the overall numerical approach developed and implemented in EUROPLEXUS fast dynamics software [7].

\section{PRESENTATION OF THE DEM/FEM MODELLING FRAMEWORK}

\subsection{Discrete element model for plain concrete}

The DEM considered herein is based on cohesive and contact interactions linking discrete elements, which are rigid spheres of different sizes and masses [8,9]. DE mesh constitutes a disordered polydisperse assembly generated by an original geometric algorithm able to fill with spheres of varying sizes a given tetrahedral mesh built for the studied concrete structure [10].

The consistency of the DEM used was evaluated through an exhaustive numerical study conducted on several DE samples [11]. Furthermore, the minimum discretization needed for a DEM sample to reproduce the macroscopic behaviour of concrete was determined.

To take into account the increase of resistance of concrete under high-rate loading, the local strain rate dependency is introduced in the model, enabling it to reproduce correctly the experimental strain rate sensitivity. An infinite impulse response filtering is applied to the velocity before calculating the strain rate.

\subsection{Steel-concrete bond modelling}

To be able to represent complex rebar patterns of actual engineering structures, we model the steel reinforcement through the conventional FEM with beam-like elements. To link concrete spherical discrete elements with FE rebar, a special steel-concrete bond model was devel- 
oped. Thus, the steel-concrete interface is modelled as a set of links established between a given rebar and the discrete concrete elements located in the vicinity of the rebar. Each link is equivalent to two independent nonlinear springs: one normal to the rebar, the other tangential. The behaviour of tangential spring is devised to reproduce the response of the steel-concrete bond observed in pull-out tests, whereas the behaviour of the normal spring is assumed to be brittle in tension and elasto-plastic in compression, Fig. 1.

\section{SIMULATION OF RESPONSE OF RC BEAMS UNDER SOFT AND HARD IMPACTS}

To demonstrate and validate the modelling capability of the proposed numerical approach we simulate low-velocity impacts on RC beams and compare numerical results with measured data obtained on CEA Orion drop tower $[12,13]$. In the experiments, to produce separately different failure modes, that is, bending and shear failures, beam reinforcement and loading were adapted in each case to favour soft or hard impact conditions.

\subsection{Test rig and experimental results description}

Two short concrete beams of $1.3 \mathrm{~m}$ length with a rectangular cross section (height $0.2 \mathrm{~m}$ and width $0.15 \mathrm{~m}$ ) are considered. The beams are made of ordinary concrete with compressive strength of $33 \mathrm{MPa}$ and are doubly reinforced with two $12 \mathrm{~mm}$ diameter ribbed high-yield steel rebars at the bottom of the beam and two $8 \mathrm{~mm}$ diameter ribbed high-yield steel rebars at the beam's top. Centres of rebars are located at $25 \mathrm{~mm}$ from the concrete surface. Rebars are welded to $1 \mathrm{~cm}$ thick steel plates disposed at the beams' ends to avoid excessive sliding between the rebars and the concrete during deformation. The first beam, aimed to the soft impact test, is also reinforced with six closed box-shape mild steel stirrups of $6 \mathrm{~mm}$ diameter and $20 \mathrm{~cm}$ spacing. Two steel semi-circular cylinders support the beams thus allowing their rotation. The beams span is $1 \mathrm{~m}$. To avoid rebound on the supports, the beams are held by two nylon straps for the first beam and two steel frames for the second one.

Impact loading is generated by heavy masses dropped from a dropping tower. A honeycomb buffer has been put between the rigid projectile and the beam to obtain the soft impact conditions, that is, to guarantee a more or less constant plateau for the impact force during the test. In the soft impact test, a mass of $311 \mathrm{~kg}$ was dropped from a height of $1.5 \mathrm{~m}$ to give the impact velocity of $5.42 \mathrm{~m} / \mathrm{s}$. In the hard impact test, a $103.65 \mathrm{~kg}$ mass dropped from a height of $3.5 \mathrm{~m}$ directly onto the beam generated the impact velocity of $8.3 \mathrm{~m} / \mathrm{s}$.

Tangential link

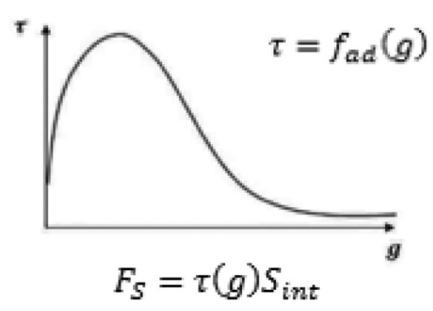

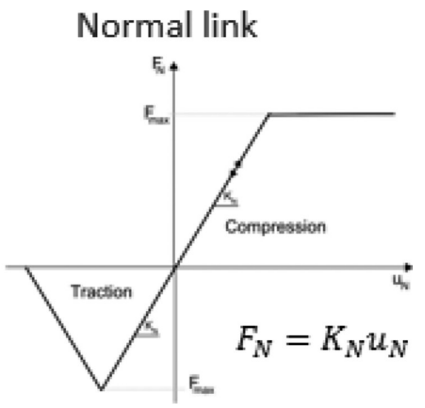

Figure 1: Interaction laws. 
For each loading case particular failure modes are generated: in the soft impact case global bending of the beam leads to the formation of a crack pattern composed of one vertical and several inclined cracks; in the hard impact case a characteristic conical shear plug delimited by oblique cracks is generated, Fig. 2 .

Figure 3 presents the time history curves issued from the soft impact test: the impact force, deflection at the beam central point and strains in steel reinforcement are shown.

\subsection{Mixed DEM/FEM modelling}

A very detailed mixed-type model of the experimental device contains an explicit mechanical modelling of the beam (including concrete by DE formulation, bending and shear steel reinforcement by FE beams, the end plates by FE hexahedra), the semi-circular cylindrical supports (FE hexahedra), the straps (FE plates), the impactor and the honeycomb buffer (both by FE hexahedra), Fig. 4.

Unilateral contact conditions are prescribed everywhere between DE and FE models as well as between the impactor and the honeycomb buffer in the soft impact case. Steel-concrete bond laws presented in the previous section are applied to describe interaction between concrete and the steel reinforcement. To study the influence of discretization refinement of concrete on the whole response of the model, two different DE meshes are built. The first DE mesh shown in Fig. 4 contains 24.677 DE elements $\left(\mathrm{R}_{\max }=1.05 \mathrm{~cm}, \mathrm{R}_{\min }=3.5 \mathrm{~mm}\right)$, whereas the second mesh is finer and contains $55.690 \mathrm{DE}$ with $\mathrm{R}_{\max }=7.9 \mathrm{~mm}, \mathrm{R}_{\min }=2.6 \mathrm{~mm}$, Fig. 5 .

\subsection{Soft impact results}

Figure 6 shows evolution of damage (which is defined herein as a ratio of remaining cohesive links over the initial number of links) in concrete for several consecutive time stations obtained with the coarse DE mesh. The final damage state can be compared with experimental crack pattern shown in Fig. 2. As can be seen the three principal cracks, a central vertical crack and two oblique cracks, are well reproduced by the simulation. Because of the mesh coarseness the secondary cracks are less developed in the calculation than in the experiment. When using the finer mesh, the crack pattern changes slightly and becomes more precise, Fig. 7. Note that the presented approach does not aim to reproduce finely and individually secondary cracks detected in the experiment. Indeed, the shape and the position of those cracks depend strongly on the local composition of concrete (aggregates location) and consequently, it will change when repeating the experiment. Thus, because of the macro modelling nature of the DE approach considered, we are seeking a global type response, that is, detecting zones of material damage and estimating the right amount of dissipated energy. In this context, it is not necessary to use highly refined mesh (and perform costly calculations) if the goal can be reached by using a moderately fine mesh.
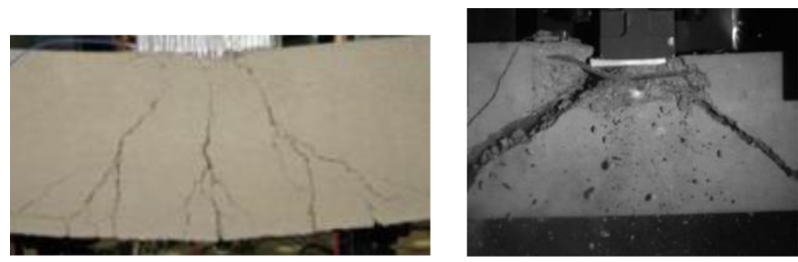

Figure 2: Bending and shear type failure modes. 


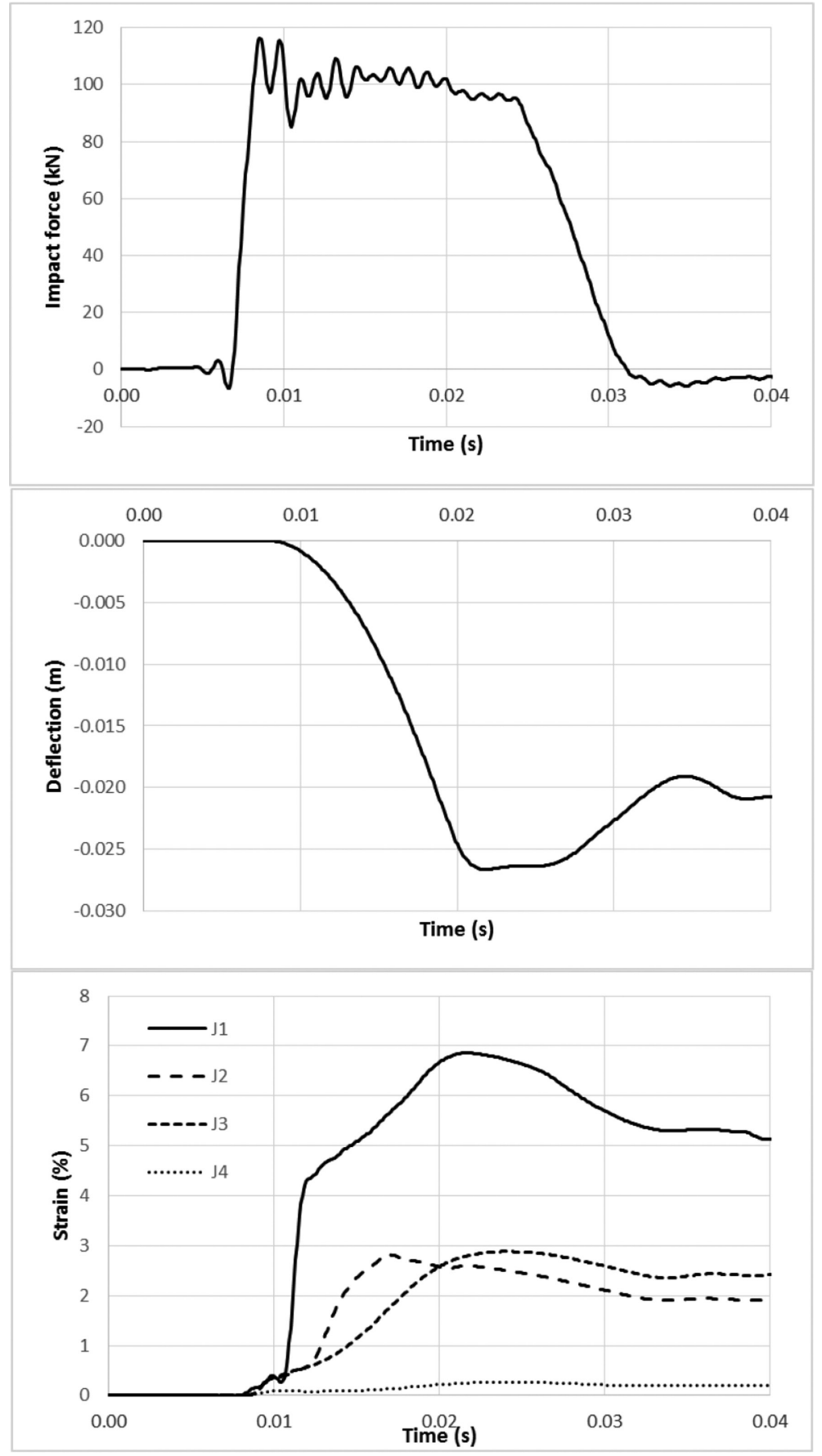

Figure 3: Experimental results from the soft impact test. 


\section{Impactor (hexa FE)}

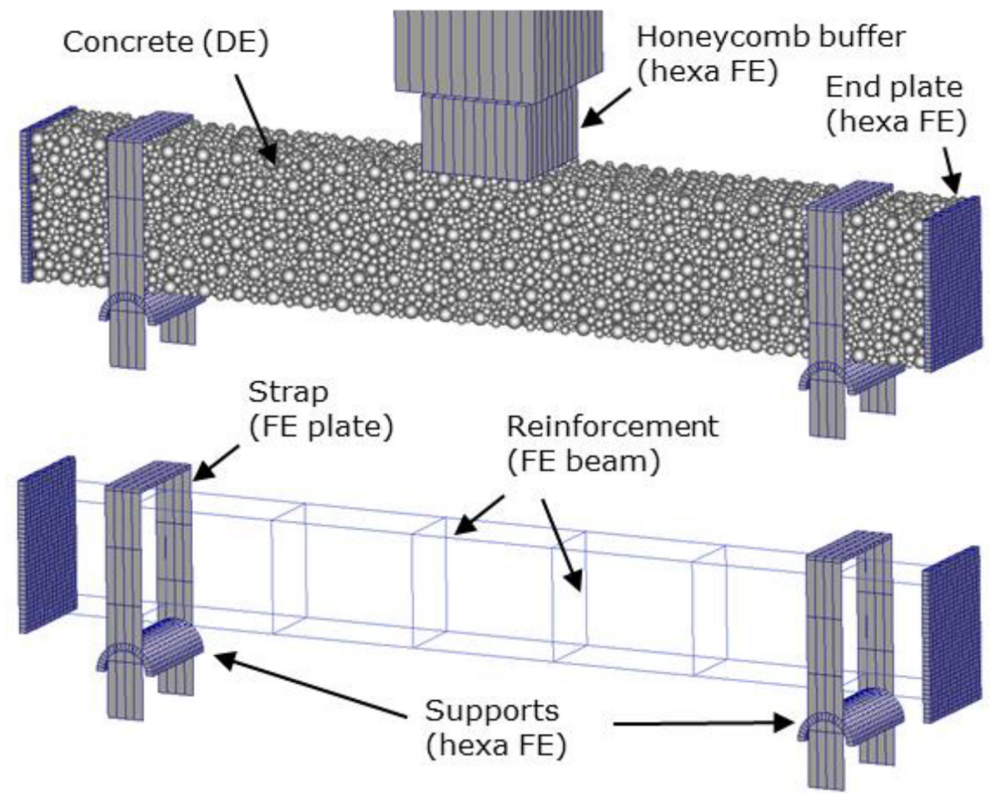

Figure 4: View of the DEM/FEM model with coarse DE mesh.

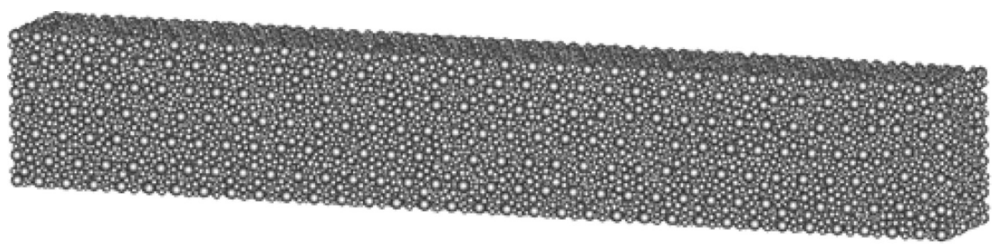

Figure 5: View of the fine DE mesh.

Thus to appreciate modelling efficiency of the DE approach, reproducibility of the global nonlinear response of the structure must be checked, namely as to the impact force, beam deflection, and strains in the steel reinforcement. Time history curves for these quantities obtained in the simulations with coarse and fine meshes are shown in Figs 8 and 9. As can be seen, they match relatively well the experimental ones (Fig. 4) for both the peak values and the general temporal trend. Moreover, residual beam deflection and residual strains in steel for some gauges (namely J1) are correctly predicted for both DE meshes considered. For gauges $\mathrm{J} 2$ and $\mathrm{J} 3$, the calculated strains are somewhat underestimated which is due to the fact that oblique cracks do not have exactly the same location as in the experiment.

\subsection{Hard impact}

In the hard impact simulations, the damage pattern is different compared to the soft impact case. Indeed, in the absence of shear reinforcement, a shear plug is formed under the impactor (Fig. 10) with the angle of the plug in conformity with the experimental observation (Fig. 3). 

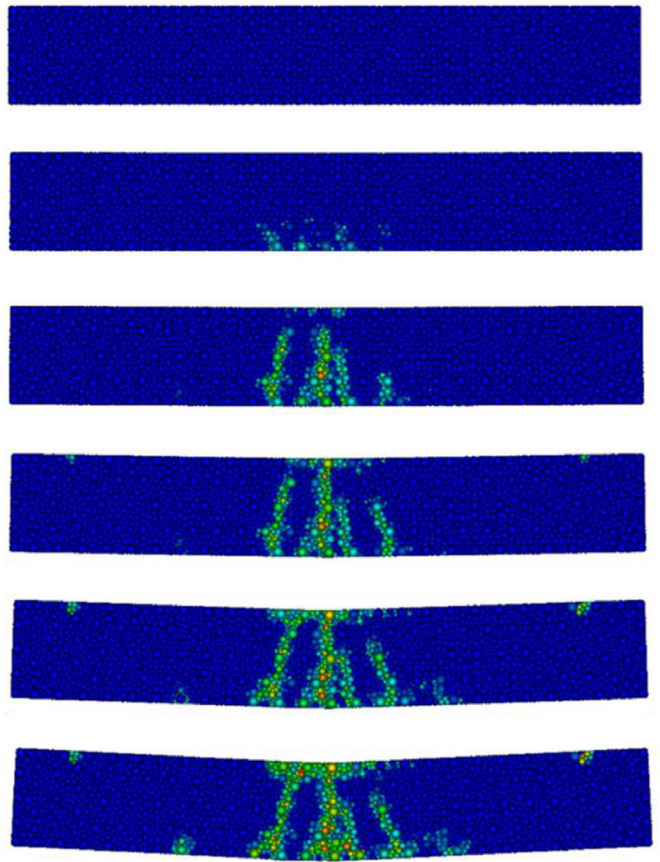

Figure 6: Evolution of damage in simulation with coarse DE mesh.
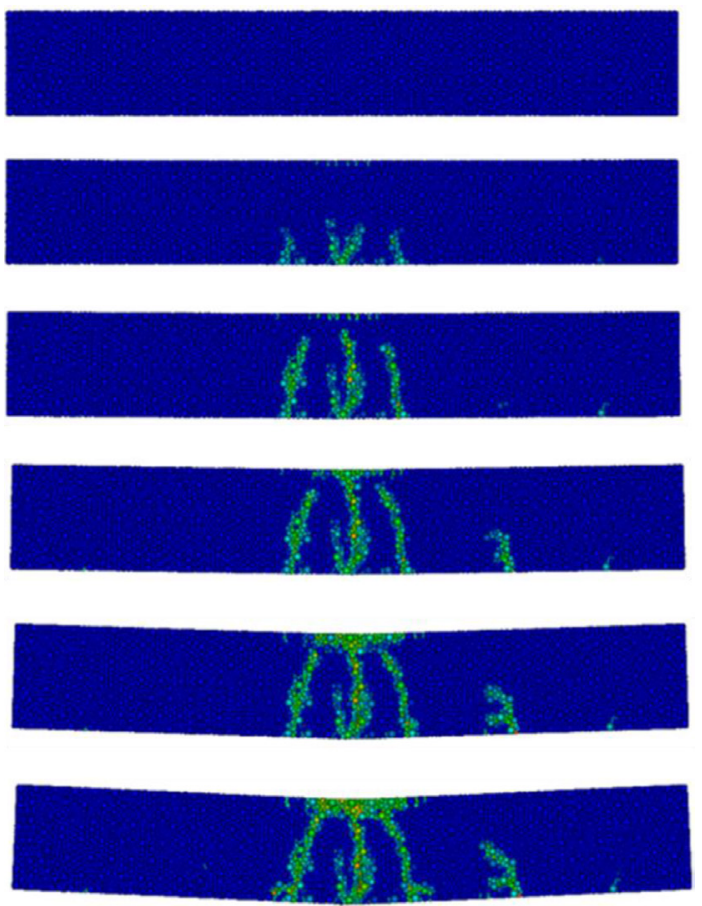

Figure 7: Evolution of damage in simulation with fine DE mesh. 




Figure 8: Results obtained with the coarse mesh. 


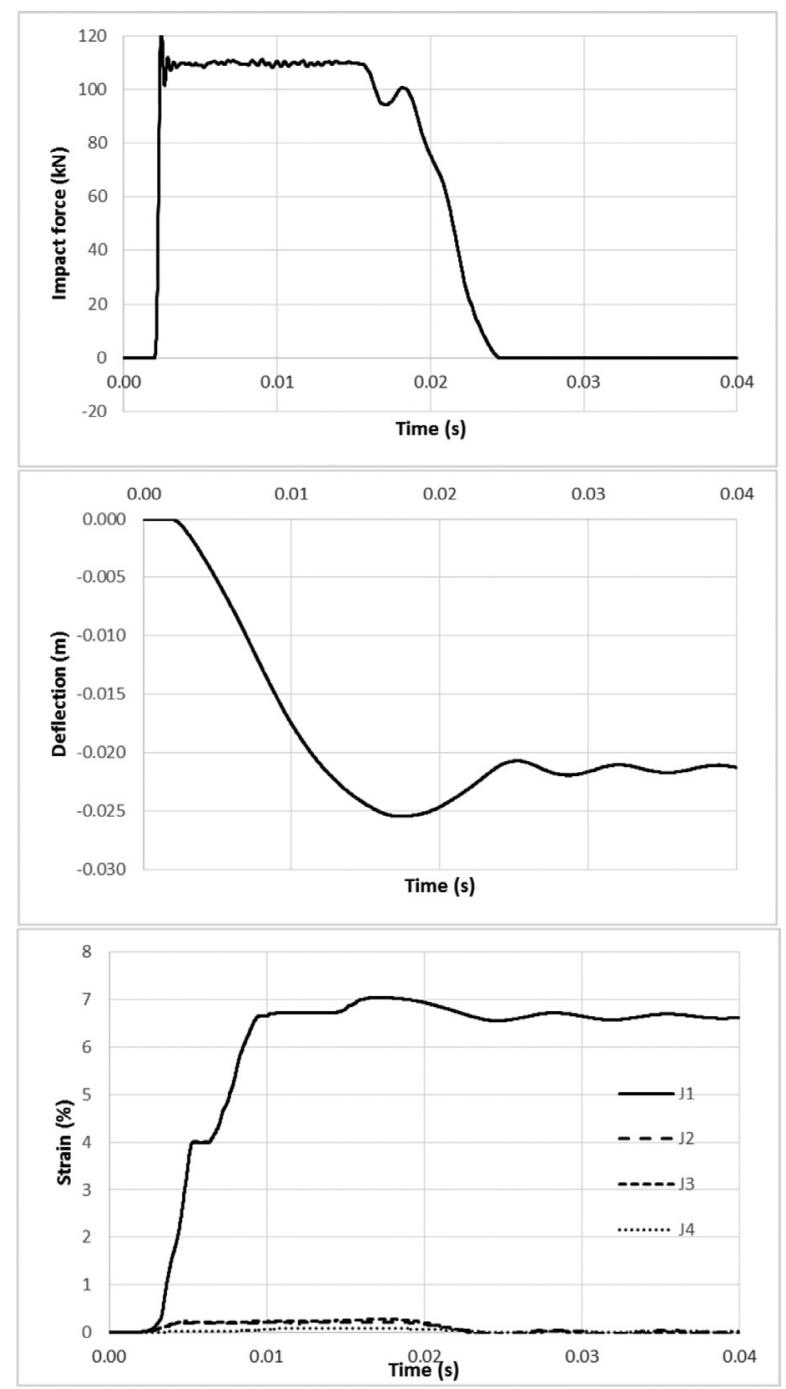

Figure 9: Results obtained with the fine mesh.

Because the diffuse damage state is difficult to interpret in terms of macro cracking, a special algorithm has been developed to detect fragments in the DE concrete model. It helps make visible material discontinuities between DE blocks created in the simulation with no common links left. These discontinuities correspond to sharp colour changes in Fig. 11. As can be seen, the damaged zone is limited by oblique cracks as observed in the experiment, see Fig. 2.

\section{CONCLUSION}

In this paper, the mixed DEM-FEM computational framework was applied to simulate advanced damage states of RC beams under soft and hard type impacts. For both soft and hard impacts, crack patterns predicted by the calculation are in general accordance with those detected in the experiment. The global nonlinear response of the RC structure is also correctly predicted, namely as to the impact force, beam deflection, and strains in steel reinforcement. 


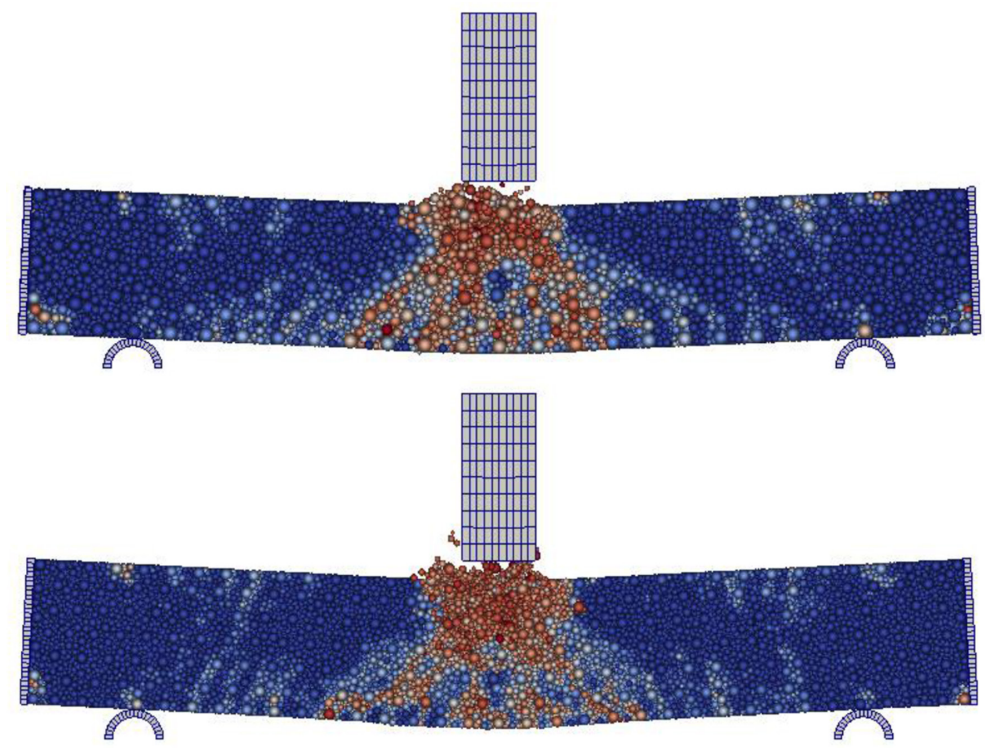

Figure 10: Final damage state obtained with the coarse and fine meshes.

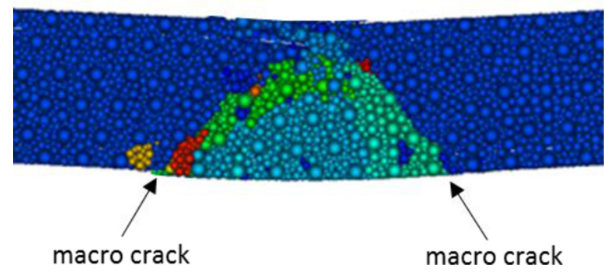

Figure 11: Concrete fragments detected in the calculation with coarse mesh.

\section{REFERENCES}

[1] Belytschko, T. \& Lin, J.I., A three-dimensional impact-penetration algorithm with erosion. Computers \& Structures, 25, pp. 95-104, 1987. http://dx.doi.org/10.1016/0045-7949(87)90220-3

[2] Camborde, F., Mariotti, C. \& Donzé, F.V., Numerical study of rock and concrete behavior by discrete element modeling. Computers and Geotechnics, 27, pp. 225-247, 2000. http://dx.doi.org/10.1016/S0266-352X(00)00013-6

[3] Hentz, S., Modélisation d'une structure en béton armé soumise à un choc par la méthode des éléments discrets. Thèse de doctorat, Université Joseph Fourier, 2003.

[4] Prado, E. \& Van Mier, J., Effect of particle structure on mode I fracture process in concrete. Engineering Fracture Mechanics, 70, pp. 1793-1807, 2003. http://dx.doi.org/10.1016/S0013-7944(03)00125-5

[5] Cusatis, G., Pelessone, D., \& Mencarelli, A., Lattice discrete particle model (LDPM) for failure behavior of concrete. I: Theory. Cement and Concrete Composites, 33, 881-890, 2011. http://dx.doi.org/10.1016/j.cemconcomp.2011.02.011 
[6] Kim, K., Bolander, J.E. \& Lim, Y.M., Failure simulation of RC structures under highly dynamic conditions using random lattice models. Computures \& Structures, 125, pp. 127-136, 2013.

http://dx.doi.org/10.1016/j.compstruc.2013.04.007

[7] EUROPLEXUS, A computer program for analysis of fast transient phenomena involving structures and fluids in interaction, available at http://www-epx.cea.fr.

[8] Rousseau, J., Modélisation numérique du comportement dynamique de structures sous impact sévère avec un couplage éléments discrets / éléments finis, Thèse de doctorat, Université Joseph Fourier: Grenoble, 2009 (in French).

[9] Hentz, S., Donzé, F.V. \& Daudeville, L., Discrete element modelling of concrete submitted to dynamic loading at high strain rate. Computers \& Structures, 82, 2509-2524, 2004.

http://dx.doi.org/10.1016/j.compstruc.2004.05.016

[10] Jerier, J-F., Richefeu, V., Imbault, D. \& Donzé, F.V., Packing spherical discrete elements for large scale simulations. Computer Methods in Applied Mechanics and Engineering, 199(25-28), pp. 1668-1676, 2010.

http://dx.doi.org/10.1016/j.cma.2010.01.016

[11] Potapov, S., Masurel, A., Marin, P. \& Daudeville, L., Mixed DEM/FEM modeling of advanced damage in reinforced concrete structures, submitted to ASCE Journal of Engineering Mechanics, EMENG-3035.

[12] Leroux, A., Modèle multiaxial d'endommagement anisotrope: gestion numérique de la rupture et application à la ruine de structures en béton armé sous impacts. PhD Thesis, Ecole normale supérieure de Cachan - ENS Cachan, 2012.

[13] Chambart, M., Endommagement anisotrope et comportement dynamique des structures en béton armé jusqu'à la ruine. PhD Thesis, Ecole normale supérieure de Cachan - ENS Cachan, 2009. 